\title{
Laplacian Eigenmaps Manifold Learning for Landmark Localization in Brain MR Images ${ }^{\star}$
}

\author{
Ricardo Guerrero, Robin Wolz, and Daniel Rueckert \\ Biomedical Image Analysis Group, Imperial College London \\ \{reg09,r.wolz, d.rueckert\}@imperial.ac.uk
}

\begin{abstract}
The identification of anatomical landmarks in medical images is an important task in registration and morphometry. Manual labeling is time consuming and prone to observer errors. We propose a manifold learning procedure, based on Laplacian Eigenmaps, that learns an embedding from patches drawn from multiple brain MR images. The position of the patches in the manifold can be used to predict the location of the landmarks via regression. New images are embedded in the manifold and the resulting coordinates are used to predict the landmark position in the new image. The output of multiple regressors is fused in a weighted fashion to boost the accuracy and robustness. We demonstrate this framework in 3D brain MR images from the ADNI database. We show an accuracy of $\sim 0.5 \mathrm{~mm}$, an increase of at least two fold when compared to traditional approaches such as registration or sliding windows.
\end{abstract}

Keywords: Manifold Learning, Laplacian Eigenmaps, Landmarks.

\section{Introduction}

The detection of landmarks is a crucial step in many medical imaging applications, including registration, shape modeling and morphometry. Approaches to landmark detection can be roughly classified into three main categories: geometric-, classification- and regression-based techniques.

Geometric-based techniques identify significant points, lines, surfaces or volumes based on features from differential geometry. In [1], 3D differential operators are used to detect salient feature points in brain Magnetic Resonance (MR) images. Using features of two- and three-point combinations, e.g. pairwise and radial distances, angles, etc., a geometrical probability approach is used in [2] to analyze the structure of 3D chromatin in interphase cell nucleii. Rosten et al. 3 used machine learning techniques to develop a high-speed corner detector, that is used to track corner features in real-time video. One of the main disadvantages with the use of geometric-based features is that geometrical saliency does not necessarily imply anatomical relevance. Another popular approach common in the computer vision community includes the use of SIFT and similar types of feature descriptors 4. Unfortunately, some of these feature detectors cannot be easily extended to $3 \mathrm{D}$.

\footnotetext{
* This project was partially funded by CONACyT, SEP and the Rabin Enzra trust.
} 
In the classification-based approach to landmark detection the general idea is to use a classifier to test an image, either exhaustively [5] or in a reduced space [6], whether a patch contains the landmark or not. In 5 the authors used a variation of AdaBoost in combination with Haar-like features to detect human faces. To locate the faces in real time in 2D images a cascade of classifiers is constructed which is applied to a window sliding over the entire image. Instead of sliding a window exhaustively across the image, Lampert et al. 6] proposed a branch and bound algorithm to reduce the search space. In [7] a probabilistic boosting tree is used to learn a discriminative model based on contextual features, with a marginal space learning strategy, for landmarks detection in cardiac MR images.

In Zhou et al. 8], a boosted regression approach is formulated for medical images. Amongst other applications, the authors test their approach for the detection and localization of anatomical structures. However, as explained in 9], the approach has two major drawbacks: First, it assumes that the output variables have a multivariate Gaussian distribution, which for real data is seldom the case. The second drawback is that the weak learner in the boosted regression is generally too weak. Therefore, the training requires the combination of a very large number of weak regressors to converge, making the training time computationally unfeasible, even for $2 \mathrm{D}$ images. In 9 the authors address these shortcomings, by adding more representational power to the weak learner.

In this work we propose a manifold learning approach that is capable of learning a low-dimensional embedding of image patches. The assumption is that the local anatomy around a particular landmark is well-represented in this embedding. We can then learn a regressor that predicts the displacements between the patch and the landmark. Patches from unseen images are mapped to the learned manifold using an out-of-sample approach and the regressor is then used to obtain an estimate of the landmark position. Finally, a consensus from the predictions made by several patches (from the same image) is reached, using a weighted average of all the estimates. The approach has been trained on a large dataset of 100 brain MR images from cognitively normal subjects (CN), patients with mild cognitive impairment (MCI) and Alzheimer's disease (AD) from the Alzheimer's Disease Neuroimaging Initiative (ADN1 ${ }^{1}$ ) study database. A different set of 100 images from ADNI is used for testing the proposed approach.

\section{Method}

\subsection{Laplacian Eigenmaps Manifold Learning}

Given a set of $N_{I}$ MR brain images, we extract $N_{P}$ equally sized patches from each image in a region of interest (ROI) around a landmark. Each patch consisting of $D$ voxels is stored as an intensity vector $\mathbf{x}_{n}=\left\{\mathrm{x}_{1}, \ldots, \mathrm{x}_{D}\right\}$ in $\mathbb{R}^{D}$, and $\mathbf{X}=\left\{\mathbf{x}_{1}, \ldots, \mathbf{x}_{N}\right\}$, where $N=N_{I} \cdot N_{P}$. Our aim is to learn the underlying manifold in $\mathbb{R}^{d}(d \ll D)$ that represents the relationship between patches in the vicinity of a given landmark. We intend to learn a manifold that can be used

${ }^{1}$ www.loni.ucla.edu/ADNI 
to predict the displacement $\Delta_{n}=\left\{\delta_{x}, \delta_{y}, \delta_{z}\right\}$ between the center of any patch and a landmark. Manifold learning offers a powerful approach to find a representation of images that facilitates the application of machine learning techniques such as regression. Since the patches lie on or near to a non-linear manifold, the Euclidean distance between patches in the original space is not necesarilly meaningful and cannot be used for regression. After uncovering the manifold structure in the data, the Euclidean distance in the embedded space provides a more meaningful approximation of the geodesic distance in the original space.

Laplacian Eigenmaps can be used to find a low-dimensional representation of the data $f: \mathbf{X} \rightarrow \mathbf{Y}, \mathbf{y}_{i}=f\left(\mathbf{x}_{i}\right)$ while preserving the local geometric properties of the manifold [10]. Laplacian Eigenmaps uses a local neighbourhood graph to approximate geodesic distances among data points. In our work we use the Euclidean norm as a distance (similarity) metric to identify the $k$-neighbourhood around each point. From these distances a sparse neighbourhood graph $G$ is constructed. Furthermore, a weight matrix $\mathbf{W}$ assigns a value to each edge in $G$, and is computed using a Gaussian heat kernel $K\left(\mathbf{x}_{i}, \mathbf{x}_{j}\right)$ with standard deviation $\sigma$. Laplacian Eigenmaps aims to place points $\mathbf{x}_{i}$ and $\mathbf{x}_{j}$ close together in the low-dimensional space, if their weight $w_{i, j}$ is high, i.e. if they are close in the high-dimensional space. This is done by minimizing the cost function given by

$$
\phi(\mathbf{Y})=\operatorname{argmin} \sum_{i, j}\left\|\mathbf{y}_{i}-\mathbf{y}_{j}\right\|^{2} w_{i, j}
$$

under the constraint that $\mathbf{y}^{T} \mathbf{D} \mathbf{y}=1$ which removes an arbitrary scaling factor in the embedding and prevents the trivial solution where all $\mathbf{y}_{i}$ are zero. The minimization of Eq. (11) can be formulated as an eigenproblem 11], through the computation of the degree matrix $\mathbf{M}$ of $\mathbf{W}$, and the Laplacian $\mathbf{L}$, where $m_{i, i}=$ $\sum_{j} w_{i, j}$ and $\mathbf{L}=\mathbf{M}-\mathbf{W}$. Hence, the low-dimensional manifold $\mathbf{Y}$ that represents all the data points can be obtained via solving a generalized eigenproblem, $\mathbf{L} \nu=$ $\lambda \mathbf{M} \nu$, where $\nu$ and $\lambda$ are the eigenvectors and eigenvalues, and in turn the $d$ smallest (non-zero) eigenvectors $\nu$ represent the new coordinate system.

\subsection{Approximate Nearest Neighbors}

Since we are learning a manifold comprised from a relatively large number of examples (219,700, see Sec. 3), the similarity matrix $\mathbf{W}$ that needs to be calculated in Laplacian Eigenmaps is very large ( $\sim 50$ billion elements), and even though it is strictly $k$-sparse, calculating exact nearest neighbours would mean that a non-sparse matrix would need to be calculated first, in order to find the $k$ nearest neighbours and then sparsify $\mathbf{W}$; making the calculation of all the exact pairwise distances computationally unfeasible. We therefore, instead calculate approximate nearest neighbors (ANN) using a hierarchical k-means tree, which is constructed by splitting the data points into $k_{m}$ distinct regions using k-means clustering, then applying the same method recursively. The recursion stops once the number of data points in each region is below $k_{m}$ [12, as implemented in the FLANN library [13. 


\subsection{Out of Sample Extension}

For the application considered in this work, it is necessary to map new patches into the manifold in order to use the embedded coordinates to make a prediction via regression. For linear dimensionality reduction techniques like PCA this is straightforward, as they provide a projection matrix for exact transformation between the original space and the embedded space. Unfortunately, this is not the case for non-linear methods. Therefore, approximation techniques must be used. We address this problem by using an out of sample technique that employs the Nyström approximation [14, which approximates the eigenvectors of a large matrix based on the eigendecomposition of a submatrix of the large matrix. Laplacian Eigenmaps are based on an initial kernel $K$, as explained in Sec. 2.1. An equivalent, training set dependent normalized kernel, is:

$$
\tilde{K}\left(\mathrm{x}_{i}^{\prime}, \mathrm{x}_{j}\right)=\frac{1}{N} \frac{K\left(\mathrm{x}_{i}^{\prime}, \mathrm{x}_{j}\right)}{\sqrt{E_{\mathbf{X}^{\prime}}\left[K\left(\mathrm{x}_{i}^{\prime}, \mathbf{X}\right)\right] E_{\mathbf{X}}\left[K\left(\mathrm{x}_{j}, \mathbf{X}\right)\right]}},
$$

where $\mathrm{x}_{j}$ and $\mathrm{x}_{i}^{\prime}$ are points from the training $\mathbf{X}$ and test $\mathbf{X}^{\prime}$ datasets, respectively, the expectations are taken over the empirical data and $N$ is the number of training samples (see [15] for full analysis).

\subsection{Spatial Prior Probabilities}

Assuming that the brain is in some approximately known orientation and possition, a landmark's spatial location is bounded, to a certain extent, to a particular volume within the brain. Once the images are affinely registered, the possible locations of each landmark are bounded within this space. Thus, we can restrict the search for each landmark to those locations which have a non-zero probability (ROI) for the location of the landmark. We model the spatial prior probabilities of each landmark, based on the position of the landmark in the training set, using kernel (or parzen window) density estimation. This can be formulated as

$$
P(\mathrm{x})=\frac{1}{n} \sum_{i=1}^{n} \frac{1}{h_{n}^{d}} K_{p}\left(\frac{\mathrm{x}-\mathrm{x}_{i}}{h_{n}}\right)
$$

where $\mathrm{x}$ are the $3 \mathrm{D}$ voxel coordinates $(x, y, z), \mathrm{x}_{i}$ are all the landmark's coordenates, $K_{p}(\cdot)$ is the window function or the kernel in a $d$-dimensional space such that $\int_{\Re d} K_{p}(\mathrm{x}) d \mathrm{x}=1, n$ is the number of observations and $h_{n}>0$ is the bandwidth parameter that corresponds to the width of the kernel. The kernel function $K_{p}(\cdot)$ is modelled as a Gaussian function.

\subsection{Landmark Prediction}

Using the low-dimensional coordinates $\mathbf{Y}=\left\{\mathbf{y}_{1}, \ldots, \mathbf{y}_{N}\right\}$ of each patch (that came from the training set) and their corresponding displacements $\Delta_{n}$ (between the centre of the patch in image space and the position of the landmark), we 
fit a linear regressor, using $\mathbf{Y}$ as independent variables and $\Delta_{n}$ as dependant variables, to obtain an estimated displacement $\Delta_{n}^{\prime}=\left\{\delta_{x}^{\prime}, \delta_{y}^{\prime}, \delta_{z}^{\prime}\right\}$.

A test dataset $\mathbf{X}^{\prime}$ is built from patches belonging to a test image's ROI. These patches are embedded in the landmark specific manifold, to obtain their low-dimentional representation $\mathbf{Y}^{\prime}$. Using the learned regressor coefficients $\beta$, an estimate of the displacement from patch $n$ to the landmark is obtained. For each test image we randomly sample 100 image patches and average the prediction results for all the patches. Since patches that predict small displacements have a higher accuracy, a weighted average was calculated, where predictions' weights are based on the magnitude of $\Delta_{n}^{\prime}$. A Gaussian kernel $K\left(\Delta_{n}^{\prime}\right)$ with $\sigma=0.65$, and mean zero was used for the weighting.

\section{$3 \quad$ Data and Results}

The images that were used to evaluate the proposed method were obtained from the ADNI database [16. In the ADNI study, brain MR images were acquired at regular intervals after an initial baseline scan from approximately 200 cognitively normal older subjects (CN), 400 subjects with mild cognitive impairment (MCI), and 200 subjects with early Alzheimers disease (AD). In this work, we used a subset of $1.5 \mathrm{~T}$ T1-weighted baseline images of 100 randomly chosen subjects for training and another 100 randomly chosen subjects for testing. In both (training and testing) datasets there are 24 AD, 48 MCI and 28 healthy subjects, to faithfully represent the full ADNI dataset. All brain images were skull stripped, affinely aligned to the MNI space and normalized using linear intensity rescaling.

The high-dimensional training set $\mathbf{X}=\left\{\mathbf{x}_{1}, \ldots, \mathbf{x}_{N}\right\}$, is obtained by collecting $3 \mathrm{D}$ image cubic patches of $21^{3}$ voxels around a regular grid that is centred at the landmark, from 100 different brain MR images. The grid has a spacing of 3 voxels and a displacement of \pm 18 voxels in each dimension. This volume is chosen so that it includes the non-zero probability volume obtained from the PDF estimation. For each image, we exhaustively sample from this grid: For each image in the training set we sample $13^{3}$ (2197) patches. Doing this for the 100 images in the trainings set and rearranging them so that each patch is represented as column vector (with $21^{3}$ values) yields a 219,700 by $9161(N, D)$ matrix that contains all the patches, from all the training images, around the landmark in question. For both the training and testing datasets a total 20 landmarks (table 1) were manually selected by an expert observer using 3 orthogonal views.

From the training set $\mathbf{X}$ we learn the underlying low-dimensional manifold, using Laplacian eigenmaps (Sec. 2.1). The parameters $k$ (nearest neighbors in the neighborhood graph), $d$ (the output dimensionality of the data) and $\sigma$ from the Gaussian heat kernel, were empirically set to 50, 200 and 1, respectively. The parameter $k$ was chosen to yield a fully connected neighbourhood graph, ensuring that all distances from the landmark were equally represented. The coefficient of determination, $R^{2}$, for the linear regression was used as an indicative to determine the final dimensionality $d$ of $\mathbf{Y}$, with values of around 0.9 obtained for 200 dimensions. Finally, tuning the parameter $\sigma$ shows little improvement. 

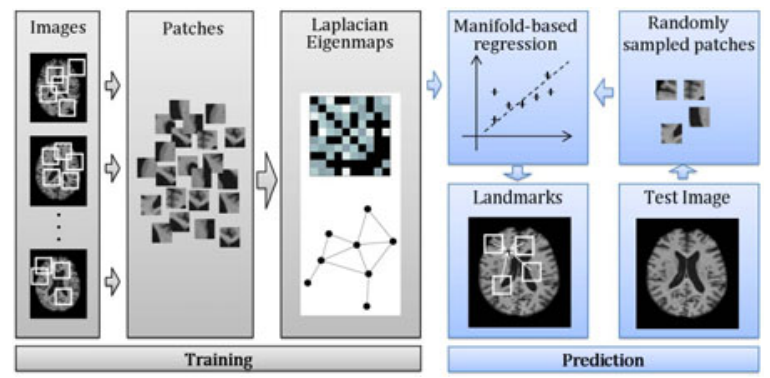

Fig. 1. Diagram of method's training and testing steps

Similarly, for each new test image, we took 100 patches at random locations, within a non-zero probability in the PDF and not necessarily belonging to the grid used in the training. We then embed the new points (patches) into the learned low-dimensional manifold, using the technique described in Sec. 2.3, and use the regression coefficients to obtain a prediction from each point. A final landmark prediction for each image is obtained, using a weighted average, as described in Sec. 2.5, A diagram of the whole process is shown in Fig. 1,

Table 1 shows results of the proposed method (landmark specific manifold, LM) and two other possible approaches: a 3D adaptation of the sliding window algorithm (SW) [5], and non-rigid registration (REG). The 3D sliding window, is trained using 400 positive and 4000 negative examples (taken from the vicinity of the positives), and it is build as a monolithic 100 feature (3D Haar features) classifier. In the registration approach, a non-rigid B-spline registration algorithm (as proposed in [17]), with a final control point spacing of 5mm, was used to propagate the landmarks from the MNI template back to the baseline images, using the transformations obtained from the registration process. A five by two cross validation of the method was carried out in order to asses the results and ensure repreducibility (the average of the five tests is showned, with a varriability among tests of $\sim 0.19 \mathrm{~mm}$ ). Our methods shows its poorest performance on the lower aspect of the cerebellum (point 16), this is mainly attributed to the fact that this particular landmark is very close to the edge of the image and the surface of the brain, meaning that relatively few patches in its vicinity contain useful information. A statistical comparison, between the three classes of patients (AD, MCI and $\mathrm{CN}$ ), of the landmark positions in the training dataset and the landmark prediction error using our method offered no intra class distinction. It was also observed that landmarks with a higher location variability tend to have a higher prediction error then those with a lower variability.

\section{Discussion and Future Work}

We have proposed a method that uses Laplacian Eigenmaps to learn a lowdimensional manifold that represents local anatomy around a specific landmark 
Table 1. Accuracy of the proposed method on the ADNI database, for the Splenium of corpus callosum (outer aspect, inferior tip and inner aspect $(1,2,3)$ ), Genu of corpus callosum (outer and inner aspect $(4,5)$ ), Superior and inferior aspect of pons $(6,7)$, Superior and inferior aspect cerebellum $(8,16)$, Fourth ventricle (9), Putamen posterior and anterior $(10,11)$ (left), $(12,13)$ (right), Anterior and posterior commissure $(14,15)$, Anterior tip of lateral ventricle (left and right) $(17,18)$, Inferior tip of lateral ventricle (left and right) $(19,20)$.

\begin{tabular}{c||c|c|c||c|c|c}
\hline \multicolumn{1}{l|}{} & \multicolumn{3}{c||}{ Mean } & \multicolumn{3}{c}{ Std. dev. } \\
\hline $\mathbf{L}$ & SW & ML & REG & SW & ML & REG \\
\hline \hline 1 & 1.75 & 0.75 & 3.95 & 1.04 & 0.31 & 1.43 \\
\hline 2 & 1.46 & 0.52 & 2.1 & 0.75 & 0.23 & 0.89 \\
\hline 3 & 1.81 & 0.51 & 2.31 & 0.99 & 0.25 & 1.31 \\
\hline 4 & 1.58 & 0.55 & 1.73 & 1.09 & 0.24 & 1.01 \\
\hline 5 & 1.28 & 0.52 & 1.47 & 0.67 & 0.25 & 0.64 \\
\hline 6 & 1.22 & 0.52 & 2.79 & 0.63 & 0.26 & 1.26 \\
\hline 7 & 1.86 & 0.59 & 1.7 & 0.92 & 0.25 & 0.85 \\
\hline 8 & 2.27 & 0.7 & 2.99 & 1.71 & 0.33 & 1.64 \\
\hline 9 & 1.09 & 0.48 & 5.57 & 0.65 & 0.22 & 2.7 \\
\hline 10 & 2.21 & 0.54 & 4.36 & 1.22 & 0.21 & 1.81 \\
\hline \hline
\end{tabular}

\begin{tabular}{c||c|c|c||c|c|c}
\hline \multicolumn{1}{l||}{} & \multicolumn{3}{c||}{ Mean } & \multicolumn{3}{c}{ Std. dev. } \\
\hline $\mathbf{L}$ & SW & ML & REG & SW & ML & REG \\
\hline \hline 11 & 1.86 & 0.53 & 2.48 & 1.13 & 0.25 & 1.29 \\
\hline 12 & 2.2 & 0.49 & 3.53 & 1.22 & 0.22 & 1.78 \\
\hline 13 & 2.31 & 0.53 & 2.79 & 1.61 & 0.26 & 1.43 \\
\hline 14 & 1.27 & 0.45 & 1.05 & 0.72 & 0.22 & 0.42 \\
\hline 15 & 0.79 & 0.46 & 1.85 & 0.6 & 0.2 & 0.48 \\
\hline 16 & 2.13 & 1.08 & 3.71 & 1.69 & 0.43 & 1.68 \\
\hline 17 & 1.86 & 0.62 & 3.67 & 1.14 & 0.33 & 1.72 \\
\hline 18 & 1.84 & 0.62 & 3.65 & 1.2 & 0.4 & 1.73 \\
\hline 19 & 2.28 & 0.57 & 4.44 & 1.42 & 0.3 & 2.07 \\
\hline 20 & 2.18 & 0.54 & 4.01 & 1.18 & 0.28 & 1.79 \\
\hline \hline
\end{tabular}

in brain MR images. The landmark specific low-dimensional manifolds were learned using image patches (around the vicinity of the landmark) from 100 brain MR images belonging to the ADNI dataset. Prior knowledge of the spatial distribution of the landmarks was used to reduce the search space. Our results show that the proposed method significantly outperforms a $3 \mathrm{D}$ sliding window, B-spline registration and a naive average, in the landmark localization task.

Anatomical landmarks of the brain have a constrained position within the brain and to the relative position of other landmarks. The inferior tip, inner and outer aspect of the splenium of the corpus callosum, are part of the same anatomical structure, so their locations with respect to each other is strongly correlated. A graphical model of the joint spatial distribution probabilities of related landmarks, in the form of a Markov random field, could be used to represent spatial dependencies. Joint spatial probabilities between related landmarks can be modeled as a continuous multivariate Gaussian. The most probable configuration of all the landmarks, according to the graphical model, where the marginal spatial probabilities of the landmarks (possibly a Gaussian fit to the output from all the predictions made from the proposed method or a SW approach) could act as a weight on the joint spatial probabilities, would yield sub-pixel accuracy.

Other possibilities for improvement could be the use of more powerful regression techniques such as support vector regression or a multiple output regression. An interesting path to explore, would be to learn a manifold of the whole brain, this would enable to locate any landmark (or pseudo landmark) within the brain, allowing to determine the position of hundreds or thousands of pseudo landmarks in every new image, allowing for a good fast first stage registration. 


\section{References}

1. Rohr, K.: On 3D differential operators for detecting point landmarks. Image and Vision Computing 15(3), 219-233 (1997)

2. Gladilin, E., Goetze, S., Mateos-Langerak, J., Van Driel, R., Rohr, K., Eils, R.: Geometrical probability approach for analysis of 3D chromatin structure in interphase cell nuclei. In: Computational Intelligence and Bioinformatics and Computational Biology, pp. 127-134 (2007)

3. Rosten, E., Drummond, T.: Machine learning for high-speed corner detection. In: Leonardis, A., Bischof, H., Pinz, A. (eds.) ECCV 2006. LNCS, vol. 3951, pp. 430443. Springer, Heidelberg (2006)

4. Li, J., Allinson, N.M.: A comprehensive review of current local features for computer vision. Neruocomputing 71, 1771-1787 (2008)

5. Viola, P., Jones, M.: Robust Real-time Face Detection. International Journal of Computer Vision 57, 137-154 (2004)

6. Lampert, C.H., Blaschko, M.B., Hofmann, T.: Beyond sliding windows: Object localization by efficient subwindow search. In: IEEE Conference on Computer Vision and Pattern Recognition, pp. 1-8. IEEE Computer Society, Los Alamitos (2008)

7. Lu, X., Georgescu, B., Littmann, A., Mueller, E., Comaniciu, D.: Discriminative Joint Context for Automatic Landmark Set Detection from a Single Cardiac MR Long Axis Slice. In: Ayache, N., Delingette, H., Sermesant, M. (eds.) FIMH 2009. LNCS, vol. 5528, pp. 457-465. Springer, Heidelberg (2009)

8. Zhou, S.K., Georgescu, B., Zhou, X.S., Comaniciu, D.: Image Based Regression Ussing Boosting Method. In: 10th IEEE International Conference on Computer Vision, vol. 1, pp. 541-548. IEEE Computer Society, Los Alamitos (2005)

9. Zhou, S.K., Zhou, J., Comaniciu, D.: A boosting regression approach to medical anatomy detection. In: IEEE Conference on Computer Vision and Pattern Recognition. IEEE Computer Society, Los Alamitos (2007)

10. Belkin, M., Nigoyi, P.: Laplacian Eigenmaps and spectral techniques for embedding and clustering. In: Advances in Neural Information Processing Systems 14, vol. 14, pp. 585-591. MIT Press, Cambridge (2002)

11. Anderson, W.N., Morley, T.D.: Eigenvalues of the Laplacian of a graph. Linear and Multilinear Algebra 18, 141-145 (1985)

12. Muja, M., Lowe, D.G.: Fast approximate nearest neighbors with automatic algorithm configuration. In: VISAPP International Conference on Computer Vision Theory and Applications, pp. 331-340. INSTICC Press (2009)

13. Fast Library for Approximate Nearest Neighbors, http://www.cs.ubc.ca/ mariusm/index.php/FLANN/FLANN

14. Platt, J.C.: FastMap, MetricMap, and Landmark MDS are all Nyström algorithms. In: Cowell, R.G., Ghahramani, Z. (eds.) 10th International Workshop on Artificial Intelligence and Statistics, pp. 261-268. Society for Artificial Intelligence and Statistics (2005)

15. Bengio, Y., Paiement, J.F., Vincent, P., Delalleau, O., Le Roux, N., Ouimet, M.: Out-of-sample extensions for LLE, Isomap, MDS, eigenmaps, and spectral clustering. In: Thrun, S., Saul, L., Schölkopf, L. (eds.) Advances in Neural Information Processing Systems 16, vol. 16, pp. 177-184. MIT Press, Cambridge (2004)

16. Mueller, S.G., Weiner, M.W., Thal, L.J., Petersen, R.C., Jack, C., Jagust, W., Trojanowski, J.Q., Toga, A.W., Beckett, L.: The Alzheimers Disease Neuroimaging Initiative. Neuroimaging Clinics of North America 15(4), 869-877 (2005)

17. Rueckert, D., Sonoda, L.I., Hayes, C., Hill, D.L.G., Leach, M.O., Hawkes, D.J.: Nonrigid Registration Using Free-Form Deformations: Application to Breast MR Images. IEEE Transactions on Medical Imaging 18(8), 712-721 (1999) 